\title{
Simultaneous Observations of Mesoscale Gravity Waves Over the Central US with CRRL Na Doppler Lidars and USU Temperature Mapper
}

\author{
Xian Lu ${ }^{1 *}$, Cao Chen ${ }^{1}$, Wentao Huang ${ }^{1}$, John A. Smith ${ }^{1}$, Jian Zhao ${ }^{1}$ Xinzhao Chu ${ }^{1 *}$, \\ Tao Yuan ${ }^{2}$, Pierre-Dominique Pautet ${ }^{2}$, Mike J. Taylor ${ }^{2}$ \\ ${ }^{1}$ University of Colorado Boulder, 216 UCB, CIRES, Boulder, CO 80309, USA, \\ *Email:xian.lu@colorado.edu andXinzhao.Chu@colorado.edu \\ ${ }^{2}$ Utah State University, Logan, UT, USA
}

\begin{abstract}
We present the first coordinated study of a 1-h mesoscale gravity wave event detected simultaneously by a $\mathrm{Na}$ Doppler lidar at Boulder, $\mathrm{CO}\left(40.1^{\circ} \mathrm{N}\right.$, $\left.105.2^{\circ} \mathrm{W}\right)$, and a $\mathrm{Na}$ Doppler lidar and an airglow temperature mapper (AMTM) at Logan, UT $\left(41.7^{\circ} \mathrm{N}\right.$, $111.8^{\circ} \mathrm{W}$ ) in the mesopause region on 27 Nov. 2013. The vertical and horizontal wavelengths are $\sim 16.0 \pm 0.3$ and $342.0 \pm 10.4 \mathrm{~km}$, corresponding to vertical and horizontal phase speeds of $\sim 4.4 \pm 0.1$ and $95.0 \pm 3.0 \mathrm{~m} / \mathrm{s}$, respectively. The wave propagates from Logan to Boulder with an azimuth angle of $\sim 138.1 \pm 1.7^{\circ}$ clockwise from North. A uniqueness of this study is that the 1-h wave amplitudes on vertical winds have been quantified for the first time by the STAR Na lidar at Boulder. The GW polarization relation between vertical wind and temperature is evaluated. The intrinsic period of the wave is Doppler shifted to $\sim 100$ min by a background wind of $40 \mathrm{~m} / \mathrm{s}$, which is confirmed by USU lidar wind observations. This study illustrates a great potential of combining multiple instruments to fully characterize mesoscale gravity waves and inspect their intrinsic properties.
\end{abstract}

\section{INTRODUCTION}

Atmospheric gravity waves (GWs) are ubiquitous in the Earth's atmosphere and play irreplaceable roles in its energetics, dynamics and chemistry [1]. Among them, mesoscale GWs with horizontal wavelengths of $50-500 \mathrm{~km}$ and periods of $1-4 \mathrm{~h}$, have important effects on weather systems by affecting mesoscale cloud and precipitation patterns [2]. They also play a significant role in the transport of momentum in the mesosphere and lower thermosphere (MLT) region, and affect ionospheric variability by seeding mediumscale travelling ionospheric disturbances. Physical and dynamical mechanisms capable of generating mesoscale GWs are largely related to loweratmosphere activity, such as convection, topographic forcing, geostrophic adjustment, and diabatic heating. Thus, the mesoscale GWs serve as important media for terrestrial weather to impact space variability.
Numerous techniques have been developed to detect and characterize GWs, while the coordinated studies that can fully resolve high to medium frequency and mesoscale gravity waves are relatively sparse. Almost all techniques have their own limitations and suffer the problem of observational filtering as each of them is only sensitive to certain portion of wave spectra and can capture certain part of wave characteristics [3]. Nevertheless, it is likely that multiple techniques have certain overlapping wave spectra that can be fully or marginally resolved and in this case, coordinated studies become meaningful.

This study aims at combining the three ground-based instruments (two $\mathrm{Na}$ Doppler lidars and an airglow imager) for the first case study of a 1-h mesoscale GW event over the central US and providing direct wave characterizations in the MLT region. In addition to temperatures $(T)$ and horizontal winds $(u, v)$, we provide the direct measurements of GW amplitudes in vertical winds $(w)$, which have been rarely reported in previous studies due to their small magnitudes and technical challenges to measure them. Such case study is important as it lays the foundation for statistical studies, which benefit the future investigations of wave sources and wave impacts upon the upper atmosphere.

\section{COORDINATED OBSERVATIONS}

This study was enabled by the simultaneous observations with two $\mathrm{Na}$ Doppler lidars of the Consortium of Resonance and Rayleigh Lidars (CRRL) at Boulder, CO [4], and at Logan, UT [5], combining with an Advanced Mesospheric Temperature Mapper (AMTM) [6] located at Bear Lake Observatory $\left(41.9^{\circ} \mathrm{N}, 111.4^{\circ} \mathrm{W}\right)$ nearby Logan. The observations were made from $\sim 1-13$ UT in the night on 27 November 2013. Under the CRRL umbrella, the Consortium Technology Center (CTC), hosted by the University of Colorado Boulder, operates a STAR Na Doppler lidar from the Table Mountain Lidar Observatory north of Boulder while the Utah State University (USU) lidar group operates a Na Doppler lidar from the USU campus in Logan. Here "STAR" stands for Student Training and Atmospheric Research. 
The STAR lidar signal levels reached over 1000 counts/shot from 80 to $115 \mathrm{~km}$ with the averaged laser power of $\sim 500 \mathrm{~mW}$ at $30 \mathrm{~Hz}$ repetition rate and a telescope primary mirror of $\sim 80 \mathrm{~cm}$ in diameter. The raw lidar data were collected in the temporal and spatial resolutions of $3 \mathrm{~s}$ and $24 \mathrm{~m}$. After binning to 5 min and $0.96 \mathrm{~km}$, the measurement uncertainties in $w$ and $T$ are $\sim 0.5 \mathrm{~m} / \mathrm{s}$ and $\sim 1 \mathrm{~K}$ near the Na layer peak. The USU lidar was operating in a two-beam setup with one pointing $30^{\circ}$ north and the other one pointing $20^{\circ}$ east. For $5 \mathrm{~min} \times 1 \mathrm{~km}$ resolution, the uncertainty near the Na peak was $\sim 3-6 \mathrm{~K}$ in $T$, and varied between $2-8$ $\mathrm{m} / \mathrm{s}$ in the Line-Of -Sight (LOS) wind.

The AMTM is an infrared digital imaging system that measures selected emission lines in the mesospheric $\mathrm{OH}(3,1)$ band to create intensity and temperature maps of the mesosphere around $87 \mathrm{~km}$. The raw temperature data are obtained with a spatial resolution of $\sim 0.5 \mathrm{~km}$ and a temporal resolution of $\sim 0.5 \mathrm{~min}$, with an uncertainty of $+/-1 \mathrm{~K}$ in temperature. Two temperature keograms, one for South-North $(\mathrm{S}-\mathrm{N})$ direction and one for West-East (W-E) direction, are composed of the S$\mathrm{N}$ and $\mathrm{W}-\mathrm{E}$ slices cutting across the center of each raw image and proceeding with time. The horizontal ranges over the S-N and W-E directions are 120 and $150 \mathrm{~km}$, respectively. The AMTM data are also binned into 5 min to have the same temporal interval as lidar data.

\section{RESULTS}

\subsection{Salient 1-h Wave Signatures}

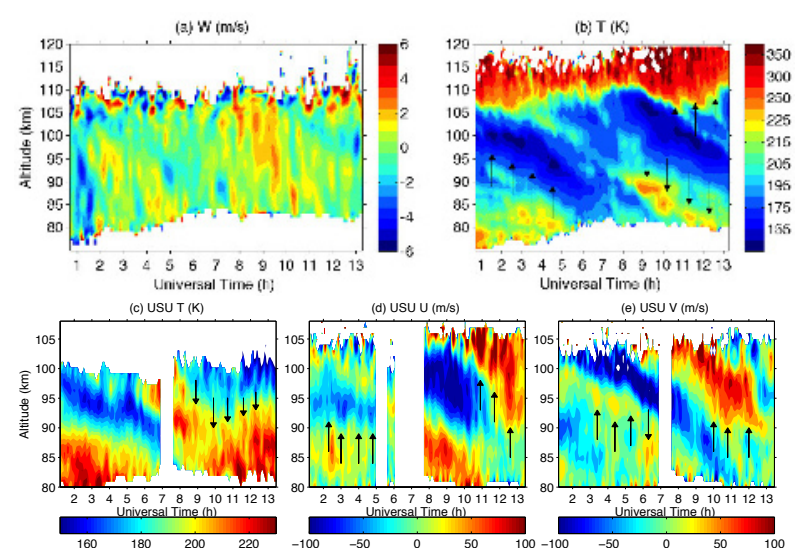

Figure 1. (a) Vertical winds and (b) temperatures from the STAR lidar at Boulder, (c)-(e) temperature and horizontal winds from USU lidar at Logan.

This study was stimulated by the signatures of shortperiod waves in the vertical winds measured by the STAR Na Doppler lidar. The most salient wave signatures in vertical winds are those with periods of 30-60 min (Figure 1a). The downward phase progressions indicate that the wave signatures are authentic and correspond to upward energy propagation. The signatures of longer period waves are visible in vertical winds as a background to these shortperiod waves. Such longer period waves or possibly tides become the prominent features in temperatures and horizontal winds (e.g., Figure 1b-e), from which higher-frequency waves are difficult to detect. Even so, the $1-\mathrm{h}$ waves are noticeable features (highlighted by black arrows in Figure 1b-e).
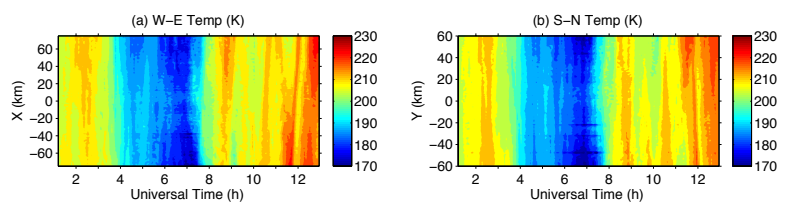

Figure 2. Raw AMTM keograms in the (a) W-E and (b) S-N directions, respectively.

Figure 2 shows the raw AMTM measurements of $\mathrm{OH}$ airglow temperatures at Logan without any filtering; however, obvious GW signatures with a period of $\sim 1 \mathrm{~h}$ are visible after $\sim 8$ UT in both directions, indicating that the presence of this wave is a robust feature.
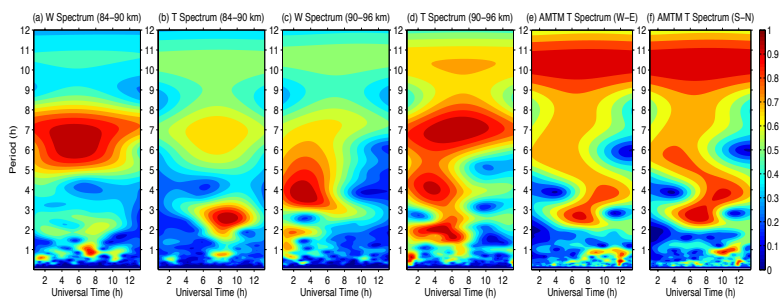

Figure 3. Normalized Morlet wavelet spectra for STAR (a) $w$ and (b) $T^{1} / T_{0}$ averaged in 84-90 km, (c) $w$ and (d) $T^{1} / T_{0}$ averaged in 90-96 km, (e) for AMTM $T^{1} / T_{0}$ in $\mathrm{W}-\mathrm{E}$ and (f) S-N directions, respectively.

To obtain perturbation fields, we subtract the nightly mean at each altitude for lidar data and at each range for AMTM. Relative temperature perturbations $\left(T^{1} / T_{0}\right)$ are further derived by dividing with nightly mean temperatures. GW Polarization relation predicts that the amplitude ratio of $w$ to $T^{\prime} / T_{0}$ is proportionally to the intrinsic frequency [7]. Thus, the $T^{1} / T_{0}$ wavelet spectra are weighted by multiplying frequency, in order to be comparable with $w$ and highlight high frequency waves. Figure 3a-d show the normalized wavelet spectra for STAR lidar, and 3e-f for the AMTM.

A rich wave spectra can be seen from the wavelet analyses. In addition to a wave component of 10-11 h which is likely the semidiurnal tide, we see strong waves with periods of 2-3 and 4-8 h. The 1-h waves persistently occur through the night while strengths vary with time. We choose a narrow passband of $0.5-$ $1.5 \mathrm{~h}$ to extract the quasi $1-\mathrm{h}$ waves due to the existence of the $2-3 \mathrm{~h}$ waves. The filtered perturbations are shown in Figure 4 for lidar data and Figure 5a-b for 
AMTM. The 1-h waves are obvious through 82-107 $\mathrm{km}$ and in the W-E and S-N directions near $87 \mathrm{~km}$.
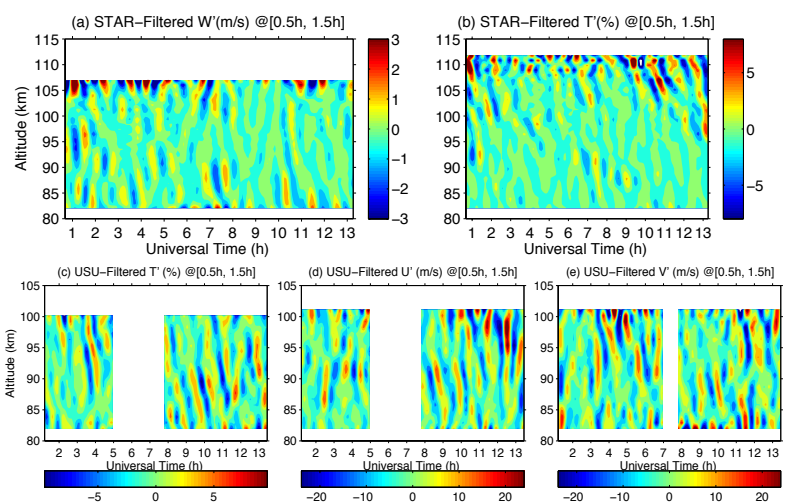

Figure 4. Perturbation fields after applying a 0.5-1.5 h bandpass filtering.
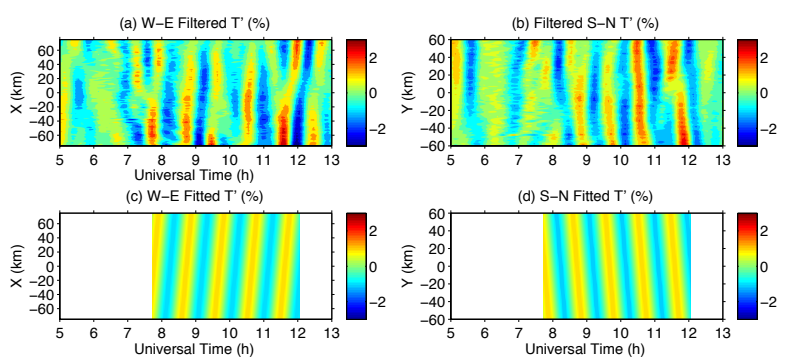

Figure 5. AMTM relative temperature perturbations filtered at $0.5-1.5 \mathrm{~h}$, and the corresponding $2 \mathrm{D}$ fittings.

\subsection{Observed Wave Characteristics}

The AMTM keograms contain the information of time versus distance, from which wave period and wavelength along either W-E or S-N direction can be inferred from the following $2 \mathrm{D}$ fitting:

$$
\frac{T^{\prime}(x, t)}{\bar{T}(x)}=A \cos \left(\frac{2 \pi}{\tau} t-\frac{2 \pi}{\lambda} x-\varphi\right)
$$

where $\tau$ and $\lambda$ are period and wavelength along the $\mathrm{W}-\mathrm{E}$ or $\mathrm{S}-\mathrm{N}$ direction, respectively. Both wavelet spectra (Figure 3) and filtered perturbations (Figure 5) illustrate strong 1-h waves between $\sim 7.7$ and 12.1 UT for AMTM, which are chosen for the fitting. The fitted periods in both directions are $\sim 0.96 \mathrm{~h}$, indicating that the same wave is identified. The wavelengths along the $\mathrm{W}-\mathrm{E}$ and S-N directions from the fittings are $511.9 \pm 21.8 \mathrm{~km}$ and $-459.6 \pm 19.8 \mathrm{~km}$, respectively. The positive sign means waves propagate eastward and the negative sign means they propagate southward.

We project such horizontal structure onto a US map centered at the states of Colorado and Utah in Figure 6. The horizontal wavelength is determined to be $342.0 \pm 10.4 \mathrm{~km}$, and the azimuth angle is $138.1^{\circ} \pm 1.7$, clockwise from North. Under such scenario, for one cycle of wave propagation, the projection onto the Logan-Boulder line would be $\sim 395.2 \mathrm{~km}$. Since the distance between these two locations is $\sim 580 \mathrm{~km}$, the 1$\mathrm{h}$ wave will take $\sim 1.47 \mathrm{~h}$ to propagate from Logan to Boulder. Thus, the phase shift between the two stations is: $\Delta \varphi=2 \pi(1.47-1) \approx 2.95 \mathrm{rad}$ at the same altitude.

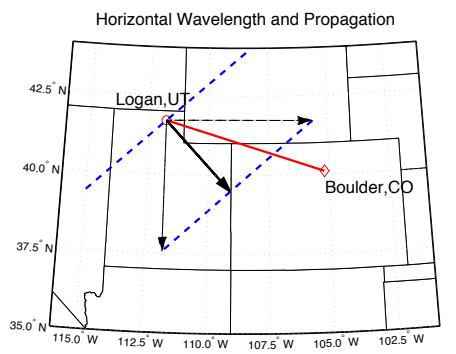

Figure 6. Geographic projection of the horizontal structure of the 1-h wave onto the US map. Thick black line with an arrow denotes the wave propagation vector $\left(\overrightarrow{k_{h}}\right)$. Dashed blue lines are two wave fronts.

Lidar measurements surpass in providing rangeresolved information of wave amplitudes and phases, and from vertical variations of phase, we can derive vertical wavelengths. Figure 7 shows the amplitudes and phases of the 1-h waves, derived from the sinusoidal fittings of the filtered perturbations [8] (Figure 4). The time window for the fitting is 7.7-10.7 UT for Logan and 9-12 UT for Boulder, which considers the time durations of relatively strong waves and the time delay caused by wave propagation.
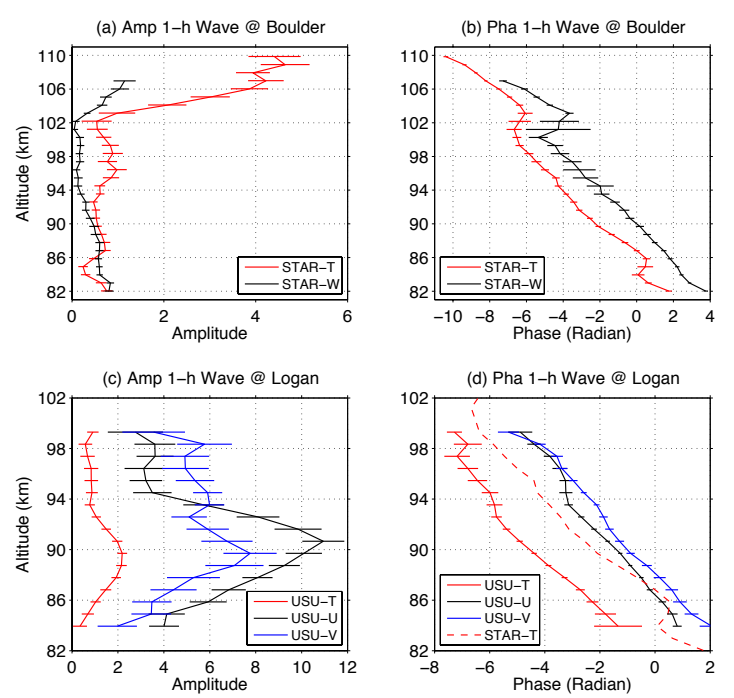

Figure 7. Amplitude and phase of the 1-h wave. The amplitude unit of $T^{\prime} / T_{0}$ is $\%$ and that of winds is $\mathrm{ms}^{-1}$.

The mean amplitudes in $T^{\prime} / T_{0}$ and $w$ at Boulder are $1.04 \%$ and $0.44 \mathrm{~m} / \mathrm{s}$, and are $1.16 \%, 6.04$ and $5.15 \mathrm{~m} / \mathrm{s}$ in $T^{\prime} / T_{0}, u$ and $v$ at Logan, respectively. The vertical wavelengths are similar among these components. The mean value is $\sim 16.0 \pm 0.3 \mathrm{~km}$. The phase difference in temperature between Logan and Boulder is about $3 \mathrm{rad}$ 
near $87 \mathrm{~km}$, which is consistent with the AMTM results. This confirms that the same wave is observed and its horizontal structure has been properly retrieved.

\subsection{Intrinsic Wave Properties}

According to the linear wave theory, the nondissipative GWs should obey the following dispersion and polarization relations [7]:

$$
\begin{gathered}
\hat{\omega}^{2}=\frac{N^{2} k_{h}^{2}+f^{2}\left(m^{2}+\frac{1}{4 H^{2}}\right)}{k_{h}^{2}+m^{2}+\frac{1}{4 H^{2}}} \\
\hat{T}=\frac{N^{2}\left(i m+\frac{1}{2 H}\right)-\frac{\hat{\omega}^{2}}{\gamma H}(\gamma-1)}{g \hat{\omega}\left(-m-\frac{i}{2 H}+\frac{i}{\gamma H}\right)} \hat{w}
\end{gathered}
$$

where $\hat{\omega}, m$, and $k_{h}$ are the wave intrinsic frequency, vertical and horizontal wavenumbers. $N$ and $H$ are buoyance frequency and scale height of the background atmosphere and equal to $\sim 0.022 \mathrm{~s}^{-1}$ and $6.1 \mathrm{~km}$ for the MLT region [9]. $f$ is the inerital frequency and $\gamma$ is the ratio of specific heats. By substituting horizontal and vertical wavelengths into $\mathrm{Eq}(2)$, the intrinsic period is calculated as $\sim 101.4 \mathrm{~min}$. Since $\hat{\omega}=\omega-\vec{k} \cdot \vec{U}$, the mean wind along the wave propagating direction should be $\sim 38.5 \mathrm{~m} / \mathrm{s}$, which is in accordance with Figure 1d-e showing that near the peak of the airglow layer $(\sim 87 \mathrm{~km})$ and from 7.7 to $12 \mathrm{UT}$, eastward zonal and southward meridional winds would have a positive projection onto the wave direction.

The polarization relation predicts that the ratio of wave amplitude in relative temperature $(\hat{T})$ to vertical wind $(\hat{w})$ is $\sim 4.5 \%$ and the phase difference is $\sim 1.69 \mathrm{rad}$. From Figure $7 b$, we derive such amplitude ratio and phase difference at each altitude and obtain their means and standard deviations as $3.4 \pm 1.9 \%$, and $1.88 \pm 0.4 \mathrm{rad}$. The theoretical predictions are within these ranges. It is expected that wave dissipations may alter the polarization relation and be responsible for the biases.

\section{CONCLUSIONS}

Simultaneous observations of two Na Doppler lidars and a temperature mapper allow us to unambigiously derive the characteristics of the 1-h mesoscale wave, shown as a dominant feature in the vertical winds of MLT region. The horizontal and vertical wavelengths of this wave are $\sim 340.0$ and $16.0 \mathrm{~km}$, corresponding to phase speeds of $\sim 95.0$ and $4.4 \mathrm{~m} / \mathrm{s}$. It propagates southeastward from Logan, UT to Boulder, CO with an azimuth angle of $\sim 138^{\circ}$. Due to the Doppler effect of background winds, its intrinsic period is shifted to $\sim 100$ min. The mean amplitude of this $1-\mathrm{h}$ wave in vertical wind is about $0.44 \mathrm{~m} / \mathrm{s}$.

A preliminary inspection of the $\mathrm{Na}$ lidar data at Boulder, $\mathrm{CO}$ has frequently revealed $0.5-2 \mathrm{~h}$ waves in vertical wind, temperature and $\mathrm{Na}$ density variations. The statistical study of their characteristics are needed to fully understand the distribution of their scales, magnitudes, and intermittency. The current study has shown a great capability of using multiple data sources to resolve the characteristics of such mesoscale and high-to-medium-frequency GWs. Future studies will be performed to link the lower-atmosphere wave sources with the observed waves in the middle atmosphere, and their coupling effects on ionospheric variabilities.

\section{ACKNOWLEDGEMENT}

The STAR lidar work was supported by NSF grants AGS-1136272 (CRRL) and AGS-1115224. X.L. was also supported by AGS-1343106 (CEDAR).

\section{REFERENCES}

[1] Holton, J. R., 1983: The influence of gravity wave breaking on the circulation of the middle atmosphere, J. Atmos. Sci., 40, 2497-2507.

[2] Zhang, F., 2004: Generation of mesoscale gravity waves in the upper-tropospheric jet-front systems, $J$. Atmos. Sci., 61, 440-457.

[3] Alexander, M. J., 1998: Interpretations of observed climatological patterns in stratospheric gravity wave variance, J. Geophys. Res., 103(D8), 8627-8640.

[4] Smith, J. A., W. Fong, B. Roberts, W. Huang and X. Chu, 2012: Very high-resolution Na Doppler lidar at Boulder, Colorado, Proc. 26th International Laser Radar Conference, 151-154.

[5] Yuan, T., et al., 2013: Coordinated investigation of summer time mid-latitude descending $\mathrm{E}$ layer (Es) perturbations using $\mathrm{Na}$ lidar, ionosonde, and meteor wind radar observations over Logan, Utah $\left(41.7^{\circ} \mathrm{N}\right.$, $\left.111.8^{\circ} \mathrm{W}\right)$, J. Geophys. Res. Atmos., 118, 1734-1746. [6] Pautet, P.-D., et al., 2014: An advanced mesospheric temperature mapper for high-latitude airglow studies, Appl. Opt., 53(26), 5934-5943.

[7] Vadas, S. L., 2013: Compressible f-plane solutions to body forces, heatings, and coolings, and application to the primary and secondary gravity waves generated by a deep convective plume, J. Geophys. Res. Space Physics, 118, 2377-2397, doi:10.1002/jgra.50163.

[8] Lu, X., et al., 2009: Gravity wave propagation and dissipation from the stratosphere to the lower thermosphere, J. Geophys. Res., 114, D11101.

[9] Lu, X., et al., 2015: Vertical evolution of potential energy density and vertical wave number spectrum of Antarctic gravity waves from 35 to $105 \mathrm{~km}$ at McMurdo (77.8 $\left.{ }^{\circ} \mathrm{S}, 166.7^{\circ} \mathrm{E}\right)$, J. Geophys. Res. Atmos., 120, doi:10.1002/2014JD022751. 